\title{
Strong convergence of hybrid Halpern iteration for Bregman totally quasi-asymptotically nonexpansive multi-valued mappings in reflexive Banach spaces with application
}

Yi Li* and Hongbo Liu

Correspondence: liyi@swust.edu.cn School of Science, Southwest University of Science and Technology, Mianyang, Sichuan 621010, P.R. China

\begin{abstract}
In this paper, Bregman totally quasi-asymptotically nonexpansive multi-valued mappings in the framework of reflexive Banach spaces are established. Under suitable limit conditions, by using the shrinking projection method introduced by Takahashi, Kubota and Takeuchi, some strong convergence theorems for hybrid Halpern's iteration for a countable family of Bregman totally quasi-asymptotically nonexpansive multi-valued mappings are proved. We apply our main results to solve classical equilibrium problems in the framework of reflexive Banach spaces. The main result presented in the paper improves and extends the corresponding result in the work by Chang (Appl. Math. Comput. 2013, doi:10.1016/j.amc.2013.11.074; Appl. Math. Comput. 228:38-48, 2014), Suthep (Comput. Math. Appl., 64:489-499, 2012), Yi Li (Fixed Point Theory Appl. 2013:197, 2013), Reich and Sabach (Nonlinear Anal. 73:122-135, 2010), Nilsrakoo and Saejung (Appl. Math. Comput. 217(14):6577-6586, 2011), Qin et al. (Appl. Math. Lett. 22:1051-1055, 2009), Wang et al. (J. Comput. Appl. Math. 235:2364-2371, 2011), Su et al. (Nonlinear Anal. 73:3890-3906, 2010) and others.
\end{abstract} MSC: 47J05; 47H09; 49J25

Keywords: Bregman totally quasi-asymptotically nonexpansive multi-valued mapping; Legendre functions; Bregman projection; fixed point; hybrid Halpern's iteration; shrinking projection method 
for all $A_{1}, A_{2} \in C B(D)$, where $d\left(x, A_{1}\right)=\inf \left\{\|x-y\|, y \in A_{1}\right\}$. The multi-valued mapping $T: D \rightarrow C B(D)$ is called nonexpansive, if

$$
H(T x, T y) \leq\|x-y\|, \quad \forall x, y \in D .
$$

An element $p \in D$ is called a fixed point of the multi-valued mapping $T: D \rightarrow N(D)$ if $p \in T(p)$. The set of fixed points of $T$ is denoted by $F(T)$.

In recent years, several types of iterative schemes have been constructed and proposed in order to get strong convergence results for finding fixed points of nonexpansive mappings in various settings. One classical and effective iteration process is defined by

$$
x_{n+1}=\alpha_{n} u+\left(1-\alpha_{n}\right) T x_{n}, \quad x_{1}, u \in D,
$$

where $\alpha_{n} \in(0,1)$. This method was introduced in 1967 by Halpern [10] and is often called Halpern's iteration. In fact, he proved, in a real Hilbert space, the strong convergence of $\left\{x_{n}\right\}$ to a fixed point of the nonexpansive mapping $T$, where $\alpha_{n}=n^{-a}, a \in(0,1)$.

Because of the simple construction, Halpern's iteration is widely used to approximate fixed points of nonexpansive mappings and other classes of nonlinear mappings by mathematicians in different styles [3-42]. In particular, some strong convergence theorems for resolvents of accretive operators in Banach spaces were proved by Reich [11], and he also extended the result of Halpern from Hilbert spaces to uniformly smooth Banach spaces in [12]. In 2012, Halpern's iteration for Bregman strongly nonexpansive mappings in reflexive Banach spaces was introduced and a strong convergence theorem for Bregman strongly nonexpansive mappings by Halpern's iteration in the framework of reflexive Banach spaces was proved. Recently, a strong convergence theorem for Bregman strongly multi-valued nonexpansive mappings as regards Halpern's iteration in the framework of reflexive Banach spaces was proved by Chang [1, 2], Suthep [3] and Li [4].

The purpose of our work is to introduce a modified Halpern iteration for a countable family of Bregman totally quasi-asymptotically nonexpansive multi-valued mappings in the framework of reflexive Banach spaces, and to prove strong convergence theorems for these iterations under suitable limit conditions by using the shrinking projection method. We use our results to solve equilibrium problems in the framework of reflexive Banach spaces. The main results presented in the paper improve and extend the corresponding results in the work by Chang [1, 2], Suthep [3], Li [4], and others.

\section{Preliminaries}

In this section, we recall some basic definitions and results which will be used in the following.

Let $X$ be a real reflexive Banach space with a norm $\|\cdot\|$, and let $X^{*}$ be the dual space of $X$. Let $f: X \rightarrow(-\infty,+\infty]$ be a proper, lower semi-continuous, and convex function. We denote by $\operatorname{dom} f=\{x \in X: f(x)<+\infty\}$ the domain of $f$.

Let $x \in \operatorname{int} \operatorname{dom} f$. The subdifferential of $f$ at $x$ is the convex set defined by

$$
\partial f(x)=\left\{x^{*} \in X^{*}: f(x)+\left\langle x^{*}, y-x\right\rangle \leq f(y), \forall y \in X\right\} .
$$

The Fenchel conjugate of $f$ is the function $f^{*}: X^{*} \rightarrow(-\infty,+\infty]$ defined by

$$
f^{*}\left(x^{*}\right)=\sup \left\{\left\langle x^{*}, x\right\rangle-f(x): x \in X\right\} .
$$


We know that the Young-Fenchel inequality holds, that is,

$$
\left\langle x^{*}, x\right\rangle \leq f(x)+f^{*}\left(x^{*}\right), \quad \forall x \in X, x^{*} \in X^{*} .
$$

Furthermore, equality holds if $x^{*} \in \partial f(x)$ (see [13]). The set $\operatorname{lev}_{\leq}^{f}(r):=\{x \in X: f(x) \leq r\}$ for some $r \in \mathbb{R}$ is called a sublevel of $f$.

A function $f$ on $X$ is called coercive [14], if the sublevel sets of $f$ are bounded, or equivalently,

$$
\lim _{\|x\| \rightarrow+\infty} f(x)=+\infty
$$

A function $f$ on $X$ is said to be strongly coercive [15], if

$$
\lim _{\|x\| \rightarrow+\infty} \frac{f(x)}{\|x\|}=+\infty
$$

For any $x \in \operatorname{int} \operatorname{dom} f$ and $y \in X$, the right-hand derivative of $f$ at $x$ in the direction $y$ is defined by

$$
f^{\circ}(x, y)=\lim _{t \rightarrow 0^{+}} \frac{f(x+t y)-f(x)}{t} .
$$

The function $f$ is said to be Gâteaux differentiable at $x$, if $\lim _{t \rightarrow 0^{+}} \frac{f(x+t y)-f(x)}{t}$ exists for any $y$. In this case, $f^{\circ}(x, y)$ coincides with $\nabla f(x)$, the value of the gradient $\nabla f(x)$ of $f$ at $x$. The function $f$ is said to be Gâteaux differentiable, if it is Gâteaux differentiable for any $x \in$ int $\operatorname{dom} f$. The function $f$ is said to be Fréchet differentiable at $x$, if this limit is attained uniformly in $\|y\|=1$. Finally, $f$ is said to be uniformly Fréchet differentiable on a subset $D$ of $X$, if the limit is attained uniformly, for $x \in D$ and $\|y\|=1$. It is well known that if $f$ is Gâteaux differentiable (resp. Fréchet differentiable) on int $\operatorname{dom} f$, then $f$ is continuous and its Gâteaux derivative $\nabla f$ is norm-to-weak*, continuous (resp. continuous) on int $\operatorname{dom} f$ (see $[16,17])$.

Definition 2.1 ( $c f$. [18]) The function $f$ is said to be

(i) essentially smooth, if $\partial f$ is both locally bounded and single-valued on its domain;

(ii) essentially strictly convex, if $(\partial f)^{-1}$ is locally bounded on its domain and $f$ is strictly convex on every convex subset of dom $\partial f$;

(iii) Legendre, if it is both essentially smooth and essentially strictly convex.

Remark 2.1 ( $c f$. [19]) Let $X$ be a reflexive Banach space. Then we have

(a) $f$ is essentially smooth if and only if $f^{*}$ is essentially strictly convex;

(b) $(\partial f)^{-1}=\partial f^{*}$;

(c) $f$ is Legendre if and only if $f^{*}$ is Legendre;

(d) If $f$ is Legendre, then $\partial f$ is a bijection which satisfies $\nabla f=\left(\nabla f^{*}\right)^{-1}$, $\operatorname{ran} \nabla f=\operatorname{dom} \nabla f^{*}=\operatorname{int} \operatorname{dom} f^{*}$ and $\operatorname{ran} \nabla f^{*}=\operatorname{dom} \nabla f=\operatorname{int} \operatorname{dom} f$.

Examples of Legendre functions can be found in [30]. One important and interesting Legendre function is $\frac{1}{p}\|\cdot\|^{p}(0<p<+\infty)$ when $X$ is a smooth and strictly convex Banach 
space. In this case the gradient $\nabla f$ of $f$ is coincident with the generalized duality mapping of $X$, i.e., $\nabla f=J_{p}$. In particular, $\nabla f=I$, the identity mapping in Hilbert spaces. In this paper, we always assume that $f$ is Legendre.

The following crucial lemma was proved by Reich and Sabach [20].

Lemma 2.1 ( $c f$. [20]) If $f: X \rightarrow R$ is uniformly Fréchet differentiable and bounded on bounded subsets of $X$, then $\nabla f$ is uniformly continuous on bounded subsets of $X$ from the strong topology of $X$ to the strong topology of $X^{*}$.

Let $f: X \rightarrow(-\infty,+\infty]$ be a convex and Gâteaux differentiable function. The function $D_{f}: \operatorname{dom} f \times \operatorname{int} \operatorname{dom} f \rightarrow[0,+\infty)$ defined by

$$
D_{f}(y, x):=f(y)-f(x)-\langle\nabla f(x), y-x\rangle
$$

is called the Bregman distance with respect to $f$.

Recall that the Bregman projection [21] of $x \in \operatorname{int} \operatorname{dom} f$ onto a nonempty, closed, and convex set $D \subset \operatorname{dom} f$ is the necessarily unique vector $\operatorname{proj}_{D}^{f}(x) \in D$ (for convenience, here we use $P_{D}^{f}(x)$ for $\left.\operatorname{proj}_{D}^{f}(x)\right)$ satisfying

$$
D_{f}\left(\operatorname{proj}_{D}^{f}(x), x\right)=\inf \left\{D_{f}(y, x): y \in D\right\} .
$$

The modulus of the total convexity of $f$ at $x \in \operatorname{int} \operatorname{dom} f$ is the function $v_{f}(x, t):[0,+\infty) \rightarrow$ $[0,+\infty)$ defined by

$$
v_{f}(x, t):=\inf \left\{D_{f}(y, x): y \in \operatorname{dom} f,\|y-x\|=t\right\} .
$$

The function $f$ is called totally convex at $x$, if $v_{f}(x, t)>0$ whenever $t>0$. The function $f$ is called totally convex, if it is totally convex at any point $x \in \operatorname{int} \operatorname{dom} f$, and it is said to be totally convex on bounded sets, if $v_{f}(B, t)>0$, for any nonempty bounded subset $B$ of and $t>0$, where the modulus of the total convexity of the function $f$ on the set $B$ is the function $v_{f}: \operatorname{int} \operatorname{dom} f \times[0,+\infty) \rightarrow[0,+\infty)$ defined by

$$
v_{f}(B, t)=\inf \left\{v_{f}(x, t): x \in B \cap \operatorname{dom} f\right\} .
$$

We know that $f$ is totally convex on bounded sets if and only if $f$ is uniformly convex on bounded sets (cf. [22]).

Recall that the function $f$ is said to be sequentially consistent [22], if for any two sequences $\left\{x_{n}\right\}$ and $\left\{y_{n}\right\}$ in $X$ such that the first sequence is bounded, the following implication holds:

$$
\lim _{n \rightarrow+\infty} D_{f}\left(x_{n}, y_{n}\right)=0 \Rightarrow \lim _{n \rightarrow+\infty}\left\|x_{n}-y_{n}\right\|=0
$$

Recall that the function $f$ is called sequentially consistent, if for any two sequences $\left\{x_{n}\right\}$ and $\left\{y_{n}\right\}$ in int $\operatorname{dom} f$ and $\operatorname{dom} f$, respectively, and $\left\{x_{n}\right\}$ is bounded, $D_{f}\left(y_{n}, x\right) \rightarrow 0$, then $\| y_{n}-$ $x \| \rightarrow 0$.

The following crucial lemma was proved by Butnariu and Iusem [23]. 
Lemma 2.2 (cf. [23]) If $x \in \operatorname{int} \operatorname{dom} f$, then the following statements are equivalent:

(i) The function $f$ is totally convex at $x$.

(ii) For any sequence $\left\{y_{n}\right\} \subset \operatorname{dom} f, D_{f}\left(y_{n}, x\right) \rightarrow 0$, then $\left\|y_{n}-x\right\| \rightarrow 0$.

Definition 2.2 ( $c f$. [24]) Let $D$ be a convex subset of int $\operatorname{dom} f$ and let $T$ be a multi-valued mapping of $D$. A point $p \in D$ is called an asymptotic fixed point of $T$ if $D$ contains a sequence $\left\{x_{n}\right\}$ which converges weakly to $p$ such that $d\left(x_{n}, T x_{n}\right) \rightarrow 0($ as $n \rightarrow \infty)$.

We denote by $\hat{F}(T)$ the set of asymptotic fixed points of $T$.

Definition 2.3 A multi-valued mapping $T: D \rightarrow N(D)$ with a nonempty fixed point set is said to be:

(i) Bregman strongly nonexpansive with respect to a nonempty $\hat{F}(T)$, if

$$
D_{f}(p, z) \leq D_{f}(p, x), \quad \forall x \in D, p \in \hat{F}(T), z \in T(x)
$$

and if, whenever $\left\{x_{n}\right\} \subset D$ is bounded, $p \in \hat{F}(T)$, and $\lim _{n \rightarrow \infty}\left[D_{f}\left(p, x_{n}\right)-D_{f}\left(p, z_{n}\right)\right]=0$, then $\lim _{n \rightarrow \infty} D_{f}\left(x_{n}, z_{n}\right)=0$, where $z_{n} \in T x_{n}$.

(ii) Bregman firmly nonexpansive if

$$
\begin{aligned}
& \left\langle\nabla f\left(x^{*}\right)-\nabla f\left(y^{*}\right), x^{*}-y^{*}\right\rangle \\
& \quad \leq\left\langle\nabla f(x)-\nabla f(y), x^{*}-y^{*}\right\rangle, \quad \forall x, y \in D, x^{*} \in T x, y^{*} \in T y .
\end{aligned}
$$

(iii) Bregman quasi-asymptotically nonexpansive mapping with sequence $\left\{k_{n}\right\} \subset[1,+\infty), k_{n} \rightarrow 1($ as $n \rightarrow \infty)$, if $\hat{F}(T)=F(T) \neq \emptyset$ and

$$
D_{f}(p, z) \leq k_{n} D_{f}(p, x), \quad p \in F(T), \forall z \in T^{n} x, x \in D .
$$

(iv) Bregman totally quasi-asymptotically nonexpansive mapping with nonnegative real sequence $\left\{v_{n}\right\},\left\{\mu_{n}\right\}, v_{n}, \mu_{n} \rightarrow 0$ (as $\left.n \rightarrow \infty\right)$ and a strictly increasing continuous function $\zeta: R^{+} \rightarrow R^{+}$with $\zeta(0)=0$, if $\hat{F}(T)=F(T) \neq \emptyset$ and

$$
D_{f}(p, z) \leq D_{f}(p, x)+v_{n} \zeta\left(D_{f}(p, x)\right)+\mu_{n}, \quad p \in F(T), \forall z \in T^{n} x, x \in D .
$$

(v) Closed, if for any sequence $\left\{x_{n}\right\} \subset D$ with $x_{n} \rightarrow x \in N(D)$ and $d\left(T x_{n}, y\right) \rightarrow 0$ $(y \in D)$, then $y \in T x$.

Remark 2.2 (cf. [1]) From these definitions, it is obvious that if $\hat{F}(T)=F(T) \neq \emptyset$, then a Bregman strongly nonexpansive multi-valued mapping is a Bregman relatively nonexpansive mapping; a Bregman relatively nonexpansive multi-valued mapping is a Bregman quasi-nonexpansive multi-valued mapping; a Bregman quasi-nonexpansive multi-valued mapping is a Bregman quasi-asymptotically nonexpansive multi-valued mapping; a Bregman quasi-asymptotically nonexpansive multi-valued mapping must be a Bregman totally quasi-asymptotically nonexpansive multi-valued mapping. However, converses of these statements are not true. 
In particular, the existence and approximation of Bregman firmly nonexpansive single value mappings was studied in [24]. It is also known that if $T$ is Bregman firmly nonexpansive and $f$ is Legendre function which is bounded, uniformly Fréchet differentiable, and totally convex on bounded subsets of $X$, then $F(T)=\hat{F}(T)$ and $F(T)$ is closed and convex (cf. [24]). It also follows that every Bregman firmly nonexpansive mapping is Bregman strongly nonexpansive with respect to $F(T)=\hat{F}(T)$. The class of single-valued Bregman totally quasi-asymptotically nonexpansive mappings was introduced first in [1]. For a wealth of results concerning this class of mappings (for example, see [1], Examples 2.11-2.15 and the references therein).

Remark 2.3 Let $X$ be a uniformly smooth and uniformly convex Banach space, and $D$ is nonempty, closed, and convex subset. An operator $T: C \rightarrow N(D)$ is called a strongly relatively nonexpansive multi-valued mapping on $X$, if $\hat{F}(T) \neq \Phi$ and

$$
\phi(p, z) \leq \phi(p, x), \quad p \in \hat{F}(T), z \in T x,
$$

and, if whenever $\left\{x_{n}\right\} \subset D$ is bounded, $p \in \hat{F}(T)$, and $\lim _{n \rightarrow \infty}\left[\phi\left(p, x_{n}\right)-\phi\left(p, z_{n}\right)\right]=0$, then $\lim _{n \rightarrow \infty} \phi\left(x_{n}, z_{n}\right)=0$, where $z_{n} \in T x_{n}$ and $\phi(x, y)=\|x\|^{2}-2\langle x, J y\rangle+\|y\|^{2}$.

Now, we give an example of Bregman totally quasi-asymptotically nonexpansive multivalued mapping.

Example 2.1 (see [1], Example 2.11) Let $D$ be a unit ball in a real Hilbert space $l^{2}, f(x)=$ $\|x\|^{2}$. Since $\nabla f(y)=2 y$, the Bregman distance with respect to $f$

$$
D_{f}(x, y)=\|x\|^{2}-\|y\|^{2}-2\langle y, x-y\rangle=\|x-y\|^{2}, \quad \forall x, y \in D .
$$

Let $T: D \rightarrow N(D)$ be a multi-valued mapping defined by

$$
T:\left(x_{1}, x_{2}, \ldots\right) \rightarrow\left(0, x_{1}^{2}, a_{2} x_{2}, a_{3} x_{3}, \ldots\right) \in l^{2}, \quad \forall\left(x_{1}, x_{2}, \ldots\right) \in D
$$

where any $\left\{a_{i}\right\}$ is a sequence in $(0,1)$ such that $\prod_{i=2}^{\infty} a_{i}=\frac{1}{2}$.

It is proved in Goebal and Kirk [25] that

(i) $\|T x-T y\| \leq 2\|x-y\|, \forall x, y \in D$;

(ii) $\left\|T^{n} x-T^{n} y\right\| \leq 2 \prod_{j=2}^{n} a_{j}\|x-y\|, \forall x, y \in D, n \geq 2$.

Let $\sqrt{k_{1}}=2, \sqrt{k_{n}}=2 \prod_{j=2}^{n} a_{j}, n \geq 2$, then $\lim _{n \rightarrow \infty} k_{n}=1$. Letting $v_{n}=k_{n}-1(n \geq 2), \zeta(t)=t$ $(t \geq 0)$, and $\left\{\mu_{n}\right\}$ be a nonnegative real sequence with $\mu_{n} \rightarrow 0$, then from (i) and (ii) we have

$$
\left\|T^{n} x-T^{n} y\right\|^{2} \leq\|x-y\|^{2}+v_{n} \zeta\left(\|x-y\|^{2}\right)+\mu_{n}, \quad \forall x, y \in D .
$$

Since $D$ is a unit ball in a real Hilbert space $l^{2}$, it follows from (2.2) that $D_{f}(x, y)=\|x-y\|^{2}$, $\forall x, y \in D$. Above inequality can be written as

$$
D_{f}\left(T^{n} x, T^{n} y\right) \leq D_{f}(x, y)+v_{n} \zeta\left(D_{f}(x, y)\right)+\mu_{n}, \quad \forall x, y \in D .
$$


Again since $0 \in D$ and $0 \in F(T)$, this implies that $F(T) \neq \Phi$. From the above inequality we get

$$
D_{f}\left(p, T^{n} x\right) \leq D_{f}(p, x)+v_{n} \zeta\left(D_{f}(p, x)\right)+\mu_{n}, \quad \forall p \in F(T), x \in D .
$$

This shows that the mapping $T$ defined as above is a Bregman total quasi-asymptotically nonexpansive multi-valued mapping.

Let $D$ be a nonempty, closed, and convex subset of $X$. Let $f: X \rightarrow \mathbb{R}$ be a Gâteaux differentiable and totally convex function and $x \in X$. It is well known from [22] that $z=P_{D}^{f}(x)$ if and only if

$$
\langle\nabla f(x)-\nabla f(z), y-z\rangle \leq 0, \quad \forall y \in D
$$

We also know the following characterization:

$$
D_{f}\left(y, P_{D}^{f}(x)\right)+D_{f}\left(P_{D}^{f}(x), x\right) \leq D_{f}(y, x), \quad \forall x, y \in D .
$$

Let $f: X \rightarrow \mathbb{R}$ be a convex, Legendre and Gâteaux differentiable function. Following [31] and [32], we make use of the function $V_{f}: X \times X^{*} \rightarrow[0,+\infty)$ associated with $f$, which is defined by

$$
V_{f}\left(x, x^{*}\right)=f(x)+f^{*}\left(x^{*}\right)-\left\langle x, x^{*}\right\rangle, \quad \forall x \in X, x^{*} \in X^{*} .
$$

Then $V_{f}$ is nonnegative and $V_{f}\left(x, x^{*}\right)=D_{f}\left(x, \nabla f^{*}\left(x^{*}\right)\right)$ for all $x \in X$ and $x^{*} \in X^{*}$. Moreover, by the subdifferential inequality (see [26], Proposition 1(iii), p.1047),

$$
V_{f}\left(x, x^{*}\right)+\left\langle y^{*}, \nabla f^{*}\left(x^{*}\right)-x\right\rangle \leq V_{f}\left(x, x^{*}+y^{*}\right), \quad \forall x \in X, x^{*}, y^{*} \in X^{*} .
$$

In addition, if $f: X \rightarrow(-\infty,+\infty]$ is a proper and lower semi-continuous function, then $f^{*}: X^{*} \rightarrow(-\infty,+\infty]$ is a proper, weak* lower semi-continuous and convex function (see [33]). Hence $V_{f}$ is convex in the second variable (see [26], Proposition 1(i), p.1047). Thus,

$$
\begin{aligned}
& D_{f}\left(z, \nabla f^{*}(t \nabla f(x)+(1-t) \nabla f(y))\right) \\
& \quad \leq t D_{f}(z, x)+(1-t) D_{f}(z, y), \quad \forall t \in(0,1), \forall x, y \in X .
\end{aligned}
$$

The properties of the Bregman projection and the relative projection operators were studied in [22] and [27].

In 2013, Yi Li and Jin-hua Zhu proved the following result, respectively.

Let $X$ be a real reflexive Banach space and let $f: X \rightarrow(-\infty,+\infty]$ be a strongly coercive Legendre function which is bounded, uniformly Fréchet differentiable, and totally convex on bounded subsets of $X$. Let $D$ be a nonempty, closed, and convex subset of int $\operatorname{dom} f$. $\alpha_{n} \in(0,1), \lim _{n \rightarrow \infty} \alpha_{n}=0$, and $0<\liminf _{n \rightarrow \infty} \beta_{n} \leq \lim \sup _{n \rightarrow \infty} \beta_{n}<1$. 
(1) (see [28]) Let $T: D \rightarrow N(D)$ be a Bregman strongly nonexpansive mapping on $X$ such that $F(T)=\hat{F}(T) \neq \emptyset$. Suppose that $u \in X$ and define the sequence $\left\{x_{n}\right\}$ by

$$
x_{1} \in D, \quad x_{n+1}=\nabla f^{*}\left(\alpha_{n} \nabla f(u)+\left(1-\alpha_{n}\right)\left(\beta_{n} \nabla f\left(x_{n}\right)+\left(1-\beta_{n}\right) \nabla f\left(T x_{n}\right)\right)\right), \quad n \geq 1 .
$$

Then $\left\{x_{n}\right\}$ strongly converges to $P_{F(T)}^{f}(u)$.

(2) (see [4]) Let $T: D \rightarrow N(D)$ be a Bregman strongly nonexpansive multi-valued mapping on $X$ such that $F(T)=\hat{F}(T) \neq \emptyset$. Suppose that $u \in X$ and define the sequence $\left\{x_{n}\right\}$ by

$$
x_{1} \in D, \quad x_{n+1}=\nabla f^{*}\left(\alpha_{n} \nabla f(u)+\left(1-\alpha_{n}\right) \nabla f\left(z_{n}\right)\right), \quad z_{n} \in T x_{n}, n \geq 1 .
$$

Then $\left\{x_{n}\right\}$ strongly converges to $P_{F(T)}^{f}(u)$.

In 2014, Chang SS proved the following result.

Theorem 2.1 ([1]) Let $X$ be a real uniformly smooth, uniformly convex, and reflexive $B a-$ nach space, $D$ be a nonempty, closed, and convex subset of $X$. Let $f: D \rightarrow(-\infty,+\infty]$ be a Legendre function which is bounded, uniformly Fréchet differentiable, and totally convex on bounded subsets of $D$ and let $\left\{T_{i}\right\}: D \rightarrow D$ be a family of closed and uniformly Bregman total quasi-asymptotically nonexpansive mappings with sequence $\left\{v_{n}\right\},\left\{\mu_{n}\right\}, v_{n}, \mu_{n} \rightarrow 0$ (as $n \rightarrow \infty$ ) and let there be a strictly increasing continuous function $\zeta: R^{+} \rightarrow R^{+}$with $\zeta(0)=0$ such that, for each $i \geq 1,\left\{T_{i}\right\}$ is uniformly $L_{i}$-Lipschitz continuous. Let $\left\{\alpha_{n}\right\}$ be a sequence in $[0,1]$ such that $\lim _{n \rightarrow \infty} \alpha_{n}=0$. Let $x_{n}$ be a sequence generated by

$$
\left\{\begin{array}{l}
x_{1} \in X \quad \text { is arbitrary; } \quad D_{1}=D, \\
y_{n, i}=\nabla f^{*}\left[\alpha_{n} \nabla f\left(x_{1}\right)+\left(1-\alpha_{n}\right) f\left(T_{i}^{n} x_{n}\right)\right], \quad i \geq 1, \\
D_{n+1}=\left\{z \in D_{n}: \sup _{i \geq 1} D_{f}\left(z, y_{n, i}\right) \leq \alpha_{n} D_{f}\left(z, x_{1}\right)+\left(1-\alpha_{n}\right) D_{f}\left(z, x_{n}\right)+\xi_{n}\right\}, \\
x_{n+1}=P_{D_{n+1}}^{f} x_{1} \quad(n=1,2, \ldots),
\end{array}\right.
$$

where $\xi_{n}=v_{n} \sup _{p \in \mathcal{F}} \zeta\left(D_{f}\left(p, x_{n}\right)\right)+\mu_{n}, \mathcal{F}=\bigcap_{i=1}^{\infty} F\left(T_{i}\right), P_{D_{n+1}}^{f}$ is the Bregman projection of $X$ onto $D_{n+1}$. If $\mathcal{F}$ is nonempty and bounded, then $\left\{x_{n}\right\}$ converges strongly to $P_{\mathcal{F}}^{f} x_{1}$.

\section{Definition 2.4}

(1) A countable family of multi-valued mappings $\left\{T_{i}: D \rightarrow N(D)\right\}_{i=1}^{\infty}$ is said to be uniformly Bregman totally quasi-asymptotically nonexpansive, if $\mathcal{F}=\bigcap_{i=1}^{\infty} F\left(T_{i}\right) \neq \varnothing$ and there exist nonnegative real sequences $\left\{v_{n}\right\},\left\{\mu_{n}\right\}, v_{n}, \mu_{n} \rightarrow 0($ as $n \rightarrow \infty)$ and a strictly increasing continuous function $\zeta: R^{+} \rightarrow R^{+}$with $\zeta(0)=0$, such that

$$
D_{f}\left(p, z_{n, i}\right) \leq D_{f}(p, x)+v_{n} \zeta\left(D_{f}(p, x)\right)+\mu_{n}, \quad p \in F(T), \forall z_{n, i} \in T_{i}^{n} x, x \in D .
$$

(2) A countable family of multi-valued mappings $\left\{T_{i}: D \rightarrow N(D)\right\}_{i=1}^{\infty}$ is said to be uniformly Bregman quasi-asymptotically nonexpansive, if $\mathcal{F}=\bigcap_{i=1}^{\infty} F\left(T_{i}\right) \neq \emptyset$ and there exist nonnegative real sequences $\left\{k_{n}\right\} \in[1,+\infty), k_{n} \rightarrow 1$ (as $\left.n \rightarrow \infty\right)$, such that

$$
D_{f}\left(p, z_{n, i}\right) \leq D_{f}(p, x)+v_{n} \zeta\left(D_{f}(p, x)\right)+\mu_{n}, \quad p \in F(T), \forall z_{n, i} \in T_{i}^{n} x, x \in D .
$$


(3) A multi-valued mapping $T: D \rightarrow N(D)$ is said to be uniformly $L$-Lipschitz continuous, if there exists a constant $L>0$ such that

$$
H\left(T^{n} x, T^{n} y\right) \leq L\|x-y\|, \quad \forall x, y \in D .
$$

Now, we improve the above results, and the following main results are obtained.

\section{Main results}

To prove our main result, we first give the following propositions.

The proof of the following result in the case of single-valued Bregman totally quasiasymptotically nonexpansive mappings was done in ([1], Lemma 2.6, and [24], Lemma 15.5). In the multi-valued case the proof is identical and therefore we will omit the exact details. The interesting reader will consult $[1,24]$.

Proposition 3.1 Let $f: X \rightarrow(-\infty,+\infty]$ be a Legendre function and let $D$ be a nonempty, closed, and convex subset of int $\operatorname{dom} f$. Let $T: D \rightarrow N(D)$ be a Bregman totally quasiasymptotically nonexpansive multi-valued mapping with respect to $f$. Then $F(T)$ is closed and convex.

Theorem 3.1 Let $X$ be a real uniformly smooth, uniformly convex, and reflexive Banach space, $D$ be a nonempty, closed, and convex subset of $X$. Let $f: D \rightarrow(-\infty,+\infty]$ be a Legendre function which is bounded, uniformly Fréchet differentiable, and totally convex on bounded subsets of $D$ and let $\left\{T_{i}\right\}: D \rightarrow N(D)$ be a family of closed and uniformly Bregman totally quasi-asymptotically nonexpansive multi-valued mappings with sequence $\left\{v_{n}\right\},\left\{\mu_{n}\right\}, v_{n}, \mu_{n} \rightarrow 0$ (as $\left.n \rightarrow \infty\right)$, and let there be a strictly increasing continuous function $\zeta: R^{+} \rightarrow R^{+}$with $\zeta(0)=0$ such that, for each $i \geq 1,\left\{T_{i}\right\}$ are uniformly $L_{i}$-Lipschitz continuous. Let $\left\{\alpha_{n}\right\}$ be a sequence in $[0,1]$ and $\left\{\beta_{n}\right\}$ be a sequence in $(0,1)$ satisfying the following conditions:

(i) $\lim _{n \rightarrow \infty} \alpha_{n}=0$;

(ii) $0<\lim _{n \rightarrow \infty} \inf \beta_{n} \leq \lim _{n \rightarrow \infty} \sup \beta_{n}<1$.

Let $x_{n}$ be a sequence generated by

$$
\left\{\begin{aligned}
x_{1} \in X \quad \text { is arbitrary; } \quad D_{1}=D, & \\
y_{n, i}= & \nabla f^{*}\left[\alpha_{n} \nabla f\left(x_{1}\right)+\left(1-\alpha_{n}\right)\left(\beta_{n} \nabla f\left(x_{n}\right)\right.\right. \\
& \left.+\left(1-\beta_{n}\right) \nabla f\left(w_{n, i}\right)\right], \quad w_{n, i} \in T_{i}^{n} x_{n}, i \geq 1, \\
D_{n+1}= & \left\{z \in D_{n}: \sup _{i \geq 1} D_{f}\left(z, y_{n, i}\right) \leq \alpha_{n} D_{f}\left(z, x_{1}\right)+\left(1-\alpha_{n}\right) D_{f}\left(z, x_{n}\right)+\xi_{n}\right\}, \\
x_{n+1}= & P_{D_{n+1}}^{f} x_{1} \quad(n=1,2, \ldots),
\end{aligned}\right.
$$

where $\xi_{n}=v_{n} \sup _{p \in \mathcal{F}} \zeta\left(D_{f}\left(p, x_{n}\right)\right)+\mu_{n}, \mathcal{F}=\bigcap_{i=1}^{\infty} F\left(T_{i}\right), P_{D_{n+1}}^{f}$ is the Bregman projection of $X$ onto $D_{n+1}$. If $\mathcal{F}$ is nonempty and bounded, then $\left\{x_{n}\right\}$ converges strongly to $P_{\mathcal{F}}^{f} x_{1}$.

Proof (I) First, we prove that $\mathcal{F}$ and $D_{n}$ are closed and convex subsets in $D$.

In fact, by Proposition 3.1 for each $i \geq 1, F\left(T_{i}\right)$ is closed and convex in $D$. Therefore $\mathcal{F}$ is a closed and convex subset in $D$. We use the assumption that $D_{1}=D$ is closed and convex. Suppose that $D_{n}$ is closed and convex for some $n \geq 1$. In view of the definition of $D_{f}(\cdot, \cdot)$, 
we have

$$
\begin{aligned}
D_{n+1}= & \left\{z \in D_{n}: \sup _{i \geq 1} D_{f}\left(z, y_{n, i}\right) \leq \alpha_{n} D_{f}\left(z, x_{1}\right)+\left(1-\alpha_{n}\right) D_{f}\left(z, x_{n}\right)+\xi_{n}\right\} \\
= & \bigcap_{i \geq 1}\left\{z \in D: \sup _{i \geq 1} D_{f}\left(z, y_{n, i}\right) \leq \alpha_{n} D_{f}\left(z, x_{1}\right)+\left(1-\alpha_{n}\right) D_{f}\left(z, x_{n}\right)+\xi_{n}\right\} \cap D_{n} \\
= & \bigcap_{i \geq 1}\left\{z \in D: \alpha_{n}\left\langle\nabla f\left(x_{1}\right), z-x_{1}\right\rangle+\left(1-\alpha_{n}\right)\left\langle\nabla f\left(x_{n}\right), z-x_{n}\right\rangle-\left\langle\nabla f\left(y_{n, i}\right), z-y_{n, i}\right\rangle\right. \\
& \left.\leq-\alpha_{n} f\left(x_{1}\right)-\left(1-\alpha_{n}\right) f\left(x_{n}\right)+f\left(y_{n, i}\right)\right\} \cap D_{n} .
\end{aligned}
$$

This shows that $D_{n+1}$ is closed and convex. The conclusions are proved.

(II) Next, we prove that $\mathcal{F} \subset D_{n}$, for all $n \geq 1$.

In fact, it is obvious that $\mathcal{F} \subset D_{1}$. Suppose that $\mathcal{F} \subset D_{n}$ for some $n \geq 1$.

Letting $\omega_{n, i}=\nabla f^{*}\left(\beta_{n} \nabla f\left(x_{n}\right)+\left(1-\beta_{n}\right) \nabla f\left(w_{n, i}\right)\right)$. Hence for any $u \in \mathcal{F} \subset D_{n}$, by (3.1), we have

$$
\begin{aligned}
D_{f}\left(u, y_{n, i}\right) & =D_{f}\left(u, \nabla f^{*}\left[\alpha_{n} \nabla f\left(x_{1}\right)+\left(1-\alpha_{n}\right) \nabla f\left(\omega_{n, i}\right)\right]\right) \\
& \leq \alpha_{n} D_{f}\left(u, x_{1}\right)+\left(1-\alpha_{n}\right) D_{f}\left(u, \omega_{n, i}\right)
\end{aligned}
$$

and

$$
\begin{aligned}
D_{f}\left(u, \omega_{n, i}\right) & =D_{f}\left(u, \nabla f^{*}\left(\beta_{n} \nabla f\left(x_{n}\right)+\left(1-\beta_{n}\right) \nabla f\left(w_{n, i}\right)\right)\right) \\
& \leq \beta_{n} D_{f}\left(u, x_{n}\right)+\left(1-\beta_{n}\right) D_{f}\left(u, w_{n, i}\right) \\
& \leq \beta_{n} D_{f}\left(u, x_{n}\right)+\left(1-\beta_{n}\right)\left\{D_{f}\left(u, x_{n}\right)+v_{n} \zeta\left[D_{f}\left(u, x_{n}\right)\right]+\mu_{n}\right\} \\
& =D_{f}\left(u, x_{n}\right)+\left(1-\beta_{n}\right) v_{n} \zeta\left[D_{f}\left(u, x_{n}\right)\right]+\left(1-\beta_{n}\right) \mu_{n} .
\end{aligned}
$$

Therefore, we have

$$
\begin{aligned}
\sup _{i \geq 1} D_{f}\left(u, y_{n, i}\right) & \leq \alpha_{n} D_{f}\left(u, x_{1}\right)+\left(1-\alpha_{n}\right)\left[D_{f}\left(u, x_{n}\right)+\left(1-\beta_{n}\right) v_{n} \zeta\left[D_{f}\left(u, x_{n}\right)\right]+\left(1-\beta_{n}\right) \mu_{n}\right] \\
& \leq \alpha_{n} D_{f}\left(u, x_{1}\right)+\left(1-\alpha_{n}\right) D_{f}\left(u, x_{n}\right)+v_{n} \sup _{p \in \mathcal{F}} \zeta\left[D_{f}\left(p, x_{n}\right)\right] \\
& =\alpha_{n} D_{f}\left(z, x_{1}\right)+\left(1-\alpha_{n}\right) D_{f}\left(z, x_{n}\right)+\xi_{n}
\end{aligned}
$$

where $\xi_{n}=v_{n} \sup _{p \in \mathcal{F}} \zeta\left(D_{f}\left(p, x_{n}\right)\right)+\mu_{n}$. This shows that $u \in \mathcal{F} \subset D_{n+1}$ and so $\mathcal{F} \subset D_{n}$. The conclusion is proved.

(III) Now we prove that $\left\{x_{n}\right\}$ converges strongly to some point $p^{*}$.

Since $x_{n}=P_{D_{n}}^{f} x_{1}$, from (2.3), we have

$$
\left\langle x_{n}-y, \nabla f\left(x_{1}\right)-\nabla f\left(x_{n}\right)\right\rangle \geq 0, \quad \forall y \in D_{n} .
$$

Again since $\mathcal{F} \subset D_{n}$, we have

$$
\left\langle x_{n}-u, \nabla f\left(x_{1}\right)-\nabla f\left(x_{n}\right)\right\rangle \geq 0, \quad \forall u \in \mathcal{F} .
$$


It follows from (2.4) that, for each $u \in \mathcal{F}$ and for each $n \geq 1$,

$$
D_{f}\left(x_{n}, x_{1}\right)=D_{f}\left(P_{D_{n}}^{f} x_{1}, x_{1}\right) \leq D_{f}\left(u, x_{1}\right)-D_{f}\left(u, x_{n}\right) \leq D_{f}\left(u, x_{1}\right)
$$

Therefore $\left\{D_{f}\left(x_{n}, x_{1}\right)\right\}$ is bounded, and so is $\left\{x_{n}\right\}$. Since $x_{n}=P_{D_{n}}^{f} x_{1}$ and $x_{n+1}=P_{D_{n+1}}^{f} x_{1} \in$ $D_{n+1} \subset D_{n}$, we have $D_{f}\left(x_{n}, x_{1}\right) \leq D_{f}\left(x_{n+1}, x_{1}\right)$. This implies that $\left\{D_{f}\left(x_{n}, x_{1}\right)\right\}$ is nondecreasing. Hence $\lim _{n \rightarrow \infty} D_{f}\left(x_{n}, x_{1}\right)$ exists.

By the construction of $\left\{D_{n}\right\}$, for any $m \geq n$, we have $D_{m} \subset D_{n}$ and $x_{m}=P_{D_{m}}^{f} x_{1} \in D_{n}$. This shows that

$$
D_{f}\left(x_{m}, x_{n}\right)=D_{f}\left(x_{m}, P_{D_{n}}^{f} x_{1}\right) \leq D_{f}\left(x_{m}, x_{1}\right)-D_{f}\left(x_{n}, x_{1}\right) \rightarrow 0 \quad(\text { as } n \rightarrow \infty) .
$$

It follows from Lemma 2.2 that $\lim _{n \rightarrow \infty}\left\|x_{m}-x_{n}\right\|=0$. Hence $\left\{x_{n}\right\}$ is a Cauchy sequence in $D$. Since $D$ is complete, without loss of generality, we can assume that $\lim _{n \rightarrow \infty} x_{n}=p^{*}$ (some point in $D$ ).

By the assumption, it is easy to see that

$$
\lim _{n \rightarrow \infty} \xi_{n}=\lim _{n \rightarrow \infty}\left[v_{n} \sup _{p \in \mathcal{F}} \zeta\left(D_{f}\left(p, x_{n}\right)\right)+\mu_{n}\right]=0 .
$$

(IV) Now we prove that $p^{*} \in \mathcal{F}$.

Since $x_{n+1} \in D_{n+1}$, from (3.1), (3.5), and (3.6), we have

$$
\sup _{i \geq 1} D_{f}\left(x_{n+1}, y_{n, i}\right) \leq \alpha_{n} D_{f}\left(x_{n+1}, x_{1}\right)+\left(1-\alpha_{n}\right) D_{f}\left(x_{n+1}, x_{n}\right)+\xi_{n} \rightarrow 0 .
$$

Since $x_{n} \rightarrow p^{*}$, it follows from (2.6) and Lemma 2.2 that for all $i \geq 1$

$$
y_{n, i} \rightarrow p^{*} \quad(\text { as } n \rightarrow \infty)
$$

Since $\left\{x_{n}\right\}$ is bounded and $\left\{T_{i}\right\}$ is a family of uniformly Bregman totally quasi-asymptotically nonexpansive multi-valued mappings, we have

$$
D_{f}\left(p, w_{n, i}\right) \leq D_{f}\left(p, x_{n}\right)+v_{n} \zeta\left[D_{f}\left(p, x_{n}\right)\right]+\mu_{n}, \quad \forall n, i \geq 1, p \in F\left(T_{i}\right), w_{n, i} \in T_{i}^{n} x_{n} .
$$

This implies that $\left\{w_{n, i}\right\}$ is uniformly bounded.

We have

$$
\begin{aligned}
\left\|\omega_{n, i}\right\| & =\left\|\nabla f^{*}\left(\beta_{n} \nabla f\left(x_{n}\right)+\left(1-\beta_{n}\right) \nabla f\left(w_{n, i}\right)\right)\right\| \\
& \leq \beta_{n}\left\|x_{n}\right\|+\left(1-\beta_{n}\right)\left\|w_{n, i}\right\| \\
& \leq\left\|x_{n}\right\|+\left\|w_{n, i}\right\|,
\end{aligned}
$$

and this implies that $\left\{\omega_{n, i}\right\}$ is also uniformly bounded.

In view of $\alpha_{n} \rightarrow 0$, from (3.1), we have

$$
\lim _{n \rightarrow \infty}\left\|\nabla f\left(y_{n, i}\right)-\nabla f\left(\omega_{n, i}\right)\right\|=\lim _{n \rightarrow \infty} \alpha_{n}\left\|\nabla f\left(x_{1}\right)-\nabla f\left(\omega_{n, i}\right)\right\|=0
$$

for each $i \geq 1$. 
Since $\nabla f^{*}$ is uniformly continuous on each bounded subset of $X^{*}$, it follows from (3.8) and (3.9) that

$$
\omega_{n, i} \rightarrow p^{*}
$$

for each $i \geq 1$. Since $\nabla f$ is uniformly continuous on each bounded subset of $X$, we have

$$
\begin{aligned}
0 & =\lim _{n \rightarrow \infty}\left\|\nabla f\left(\omega_{n, i}\right)-\nabla f\left(p^{*}\right)\right\| \\
& =\lim _{n \rightarrow \infty}\left\|\beta_{n} \nabla f\left(x_{n}\right)+\left(1-\beta_{n}\right) \nabla f\left(w_{n, i}\right)-\nabla f\left(p^{*}\right)\right\| \\
& =\lim _{n \rightarrow \infty}\left\|\beta_{n}\left(\nabla f\left(x_{n}\right)-\nabla f\left(p^{*}\right)\right)+\left(1-\beta_{n}\right)\left(\nabla f\left(w_{n, i}\right)-\nabla f\left(p^{*}\right)\right)\right\| \\
& =\lim _{n \rightarrow \infty}\left(1-\beta_{n}\right)\left\|\nabla f\left(w_{n, i}\right)-\nabla f\left(p^{*}\right)\right\| .
\end{aligned}
$$

By condition (ii), we have

$$
\lim _{n \rightarrow \infty}\left\|\nabla f\left(w_{n, i}\right)-\nabla f\left(p^{*}\right)\right\|=0 .
$$

Since $J$ is uniformly continuous, this shows that

$$
\lim _{n \rightarrow \infty} w_{n, i}=p^{*}
$$

for each $i \geq 1$. Again by the assumptions that $\left\{T_{i}\right\}: D \rightarrow D$ be uniformly $L_{i}$-Lipschitz continuous for each $i \geq 1$, thus we have

$$
\begin{aligned}
& H\left(T_{i}^{n+1} x_{n}, T_{i}^{n} x_{n}\right) \\
& \quad \leq H\left(T_{i}^{n+1} x_{n}, T_{i}^{n+1} x_{n+1}\right)+d\left(T_{i}^{n+1} x_{n+1}, x_{n+1}\right) \\
& \quad+d\left(x_{n+1}, x_{n}\right)+d\left(x_{n}, T_{i}^{n} x_{n}\right) \\
& \quad \leq\left(L_{i}+1\right) d\left(x_{n+1}, x_{n}\right)+d\left(T_{i}^{n+1} x_{n+1}, x_{n+1}\right)+d\left(x_{n}, T_{i}^{n} x_{n}\right),
\end{aligned}
$$

for each $i \geq 1$.

We get $\lim _{n \rightarrow \infty}\left\|H\left(T_{i}^{n+1} x_{n}\right)-H\left(T_{i}^{n} x_{n}\right)\right\|=0$. Since $\lim _{n \rightarrow \infty} w_{n, i}=p^{*}$ and $\lim _{n \rightarrow \infty} x_{n}=p^{*}$, we have $\lim _{n \rightarrow \infty} d\left(H\left(T_{i} T_{i}^{n} x_{n}\right), p^{*}\right)=0$.

In view of the closedness of $T_{i}$, it yields $d\left(T_{i} p^{*}, p^{*}\right)=0$. Since $p^{*} \in C, p^{*} \in T_{i} p^{*}$, i.e., for each $i \geq 1, p^{*} \in F\left(T_{i}\right)$. By the arbitrariness of $i \geq 1$, we have $p^{*} \in \mathcal{F}$.

(V) Finally we prove that $p^{*}=P_{\mathcal{F}}^{f} x_{1}$ and so $x_{n} \rightarrow P_{\mathcal{F}}^{f} x_{1}=p^{*}$.

Let $u=P_{\mathcal{F}}^{f} x_{1}$. Since $u \in \mathcal{F} \subset D_{n}$ and $x_{n}=P_{D_{n}}^{f} x_{1}$, we have $D_{f}\left(x_{n}, x_{1}\right) \leq D_{f}\left(w, x_{1}\right)$. This implies that

$$
D_{f}\left(p^{*}, x_{1}\right)=\lim _{n \rightarrow \infty} D_{f}\left(x_{n}, x_{1}\right) \leq D_{f}\left(u, x_{1}\right),
$$

which yields $p^{*}=u=P_{\mathcal{F}}^{f} x_{1}$. Therefore, $x_{n} \rightarrow P_{\mathcal{F}}^{f} x_{1}$. The proof of Theorem 3.1 is completed.

By Remark 2.2, the following corollary is obtained. 
Theorem 3.2 Let $D, X,\left\{\alpha_{n}\right\},\left\{\beta_{n}\right\}$, and $f$ be the same as in Theorem 3.1, Let $\left\{T_{i}\right\}: D \rightarrow$ $N(D)$ be a family of closed and uniformly Bregman quasi-asymptotically nonexpansive multi-valued mappings with sequence $\left\{k_{n}\right\} \subset[1,+\infty), k_{n} \rightarrow 1$ (as $\left.n \rightarrow \infty\right)$ such that, for each $i \geq 1,\left\{T_{i}\right\}$ be uniformly $L_{i}$-Lipschitz continuous. Let $\left\{\alpha_{n}\right\}$ be a sequence in $[0,1]$ and $\left\{\beta_{n}\right\}$ be a sequence in $(0,1)$ satisfying the following conditions:

(i) $\lim _{n \rightarrow \infty} \alpha_{n}=0$;

(ii) $0<\lim _{n \rightarrow \infty} \inf \beta_{n} \leq \lim _{n \rightarrow \infty} \sup \beta_{n}<1$.

Let $x_{n}$ be a sequence generated by

$$
\left\{\begin{aligned}
x_{1} \in & X \quad \text { is arbitrary; } \quad D_{1}=D, \\
y_{n, i}= & \nabla f^{*}\left[\alpha_{n} \nabla f\left(x_{1}\right)+\left(1-\alpha_{n}\right)\left(\beta_{n} \nabla f\left(x_{n}\right)\right.\right. \\
& \left.+\left(1-\beta_{n}\right) \nabla f\left(w_{n, i}\right)\right], \quad w_{n, i} \in T_{i}^{n} x_{n}, i \geq 1, \\
D_{n+1}= & \left\{z \in D_{n}: \sup _{i \geq 1} D_{f}\left(z, y_{n, i}\right) \leq \alpha_{n} D_{f}\left(z, x_{1}\right)+\left(1-\alpha_{n}\right) D_{f}\left(z, x_{n}\right)+\xi_{n}\right\}, \\
x_{n+1}= & P_{D_{n+1}}^{f} x_{1} \quad(n=1,2, \ldots),
\end{aligned}\right.
$$

where $\xi_{n}=\left(k_{n}-1\right) \sup _{p \in \mathcal{F}} \zeta\left(D_{f}\left(p, x_{n}\right)\right), \mathcal{F}=\bigcap_{i=1}^{\infty} F\left(T_{i}\right), P_{D_{n+1}}^{f}$ is the Bregman projection of $X$ onto $D_{n+1}$. If $\mathcal{F}$ is nonempty, then $\left\{x_{n}\right\}$ converges strongly to $P_{\mathcal{F}}^{f} x_{1}$.

As a direct consequence of Theorem 3.1 and Remark 2.3, we obtain the convergence result concerning Bregman totally quasi-asymptotically nonexpansive multi-valued mappings in a uniformly smooth and uniformly convex Banach space.

Theorem 3.3 Let $X$ be a uniformly smooth and uniformly convex Banach space and $J: X \rightarrow 2^{X^{*}}$ is the normalized duality mapping. Let $D$ be a nonempty, closed, and convex subset on $X$ and let $T: D \rightarrow N(D)$ be a family of closed and uniformly Bregman totally quasi-asymptotically nonexpansive multi-valued mappings with sequence $\left\{v_{n}\right\},\left\{\mu_{n}\right\}$, $v_{n}, \mu_{n} \rightarrow 0($ as $n \rightarrow \infty)$ and a strictly increasing continuous function $\zeta: R^{+} \rightarrow R^{+}$with $\zeta(0)=0$ such that, for each $i \geq 1,\left\{T_{i}\right\}$ be uniformly $L_{i}$-Lipschitz continuous. Let $\left\{\alpha_{n}\right\}$ be a sequence in $[0,1]$ and $\left\{\beta_{n}\right\}$ be a sequence in $(0,1)$ satisfying the following conditions:

(C1) $\lim _{n \rightarrow \infty} \alpha_{n}=0$ and $\sum_{n=1}^{\infty} \alpha_{n}=\infty$,

(C2) $0<\liminf _{n \rightarrow \infty} \beta_{n} \leq \lim \sup _{n \rightarrow \infty} \beta_{n}<1$.

Let $x_{n}$ be a sequence generated by

$$
\left\{\begin{array}{l}
x_{1} \in X \quad \text { is arbitrary; } \quad D_{1}=D, \\
y_{n, i}=J^{-1}\left(\alpha_{n} J x_{1}+\left(1-\alpha_{n}\right)\left(\beta_{n} J x_{n}+\left(1-\beta_{n}\right) J w_{n, i}\right), \quad w_{n, i} \in T_{i}^{n} x_{n}, i \geq 1,\right. \\
D_{n+1}=\left\{z \in D_{n}: \sup _{i \geq 1} \phi\left(z, y_{n, i}\right) \leq \alpha_{n} \phi\left(z, x_{1}\right)+\left(1-\alpha_{n}\right) \phi\left(z, x_{n}\right)+\xi_{n}\right\}, \\
x_{n+1}=\Pi_{D_{n+1}} x_{1} \quad(n=1,2, \ldots),
\end{array}\right.
$$

where $\xi_{n}=v_{n} \sup _{p \in \mathcal{F}} \zeta\left(\phi\left(p, x_{n}\right)\right)+\mu_{n}, \mathcal{F}=\bigcap_{i=1}^{\infty} F\left(T_{i}\right), \Pi_{D_{n+1}}$ is a projection of $X$ onto $D_{n+1}$. If $\mathcal{F}$ is nonempty and bounded, then $\left\{x_{n}\right\}$ converges strongly to $\Pi_{\mathcal{F}} x_{1}$.

Now, we provide examples of multi-valued mappings to which the results of the paper can be applied. 
Example 3.1 Let $D$ be a unit ball in a real Hilbert space $l^{2}, f(x)=\|x\|^{2}$. Since $\nabla f(y)=2 y$, the Bregman distance with respect to $f$

$$
D_{f}(x, y)=\|x\|^{2}-\|y\|^{2}-2\langle y, x-y\rangle=\|x-y\|^{2}, \quad \forall x, y \in D .
$$

Let $\left\{T_{i}\right\}_{i=1}^{\infty}: D \rightarrow N(D)$ be a family multi-valued mapping defined by

$$
\left\{T_{i}\right\}_{i=1}^{\infty}:\left(x_{1}^{(i)}, x_{2}^{(i)}, \ldots\right) \rightarrow\left(0,\left(x_{1}^{(i)}\right)^{2}, a_{2} x_{2}^{(i)}, a_{3} x_{3}^{(i)}, \ldots\right) \in l^{2}, \quad \forall\left(x_{1}^{(i)}, x_{2}^{(i)}, \ldots\right) \in D
$$

where any $\left\{a_{j}\right\}_{j=1}^{\infty}$ is a sequence in $(0,1)$ such that $\prod_{j=2}^{\infty} a_{j}=\frac{1}{2}$.

From Example 2.1, we know that $\left\{T_{i}\right\}_{i=1}^{\infty}$ is a family of closed and uniformly Bregman totally quasi-asymptotically nonexpansive multi-valued mappings with sequence $\left\{v_{n}\right\},\left\{\mu_{n}\right\}$, $v_{n}, \mu_{n} \rightarrow 0($ as $n \rightarrow \infty)$ and a strictly increasing continuous function $\zeta: R^{+} \rightarrow R^{+}$with $\zeta(0)=0$ such that, for each $i \geq 1,\left\{T_{i}\right\}_{i=1}^{\infty}$ is uniformly $L_{i}$-Lipschitz continuous. $\left\{\alpha_{n}\right\},\left\{\beta_{n}\right\}$ and $f$ are the same as in Theorem 3.1. Let $\left\{x_{n}\right\}$ be a sequence generated by (3.1), then $\left\{x_{n}\right\}$ converges strongly to $P_{\mathcal{F}}^{f} x_{1}$, where $\mathcal{F}=\bigcap_{i=1}^{\infty} F\left(T_{i}\right)$ is nonempty, $P_{\mathcal{F}}^{f}$ is the Bregman projection of $X$ onto $\mathcal{F}$.

\section{Application}

In order to emphasize the importance of Theorem 3.1, we illustrate an application with the following important example, which entails equilibrium problems in the framework of reflexive Banach spaces.

Let $X$ be a smooth, strictly convex, and reflexive Banach space, let $D$ be a nonempty, closed, and convex subset of $X$ and let $G: D \times D \rightarrow R$ be a bifunction satisfying the conditions: (A1) $G(x, x)=0$, for all $x \in D$; (A2) $G(x, y)+G(y, x) \leq 0$, for any $x, y \in D$; (A3) for each $x, y, z \in D, \lim _{t \rightarrow 0} G(t z+(1-t) x, y) \leq G(x, y)$; (A4) for each given $x \in D$, the function $y \longmapsto f(x, y)$ is convex and lower semicontinuous. The 'so-called' equilibrium problem for $G$ is to find a $x^{*} \in D$ such that $G\left(x^{*}, y\right) \geq 0$, for each $y \in D$. The set of its solutions is denoted by $E P(G)$.

The resolvent of a bifunction $G[5]$ is the operator $\operatorname{Res}_{G}^{f}: X \rightarrow 2^{D}$ defined by

$$
\operatorname{Res}_{G}^{f}(x)=\{z \in D, G(z, y)+\langle\nabla f(z)-\nabla f(x), y-z\rangle \geq 0, \forall y \in D\}, \quad \forall x \in X .
$$

If $f: X \rightarrow(-\infty,+\infty]$ is a strongly coercive and Gâteaux differentiable function, and $G$ satisfies conditions (A1)-(A4), then $\operatorname{dom}\left(\operatorname{Res}_{G}^{f}\right)=X$ (see [5]). We also know:

(1) $\operatorname{Res}_{G}^{f}$ is single-valued;

(2) $\operatorname{Res}_{G}^{f}$ is a Bregman firmly nonexpansive mapping, so it is a closed Bregman total quasi-asymptotically nonexpansive mapping;

(3) $F\left(\operatorname{Res}_{G}^{f}\right)=E P(G)$;

(4) $E P(G)$ is a closed and convex subset of $D$;

(5) for all $x \in X$ and for all $p \in F\left(\operatorname{Res}_{G}^{f}\right)$, we have

$$
D_{f}\left(p, \operatorname{Res}_{G}^{f}(x)\right)+D_{f}\left(\operatorname{Res}_{G}^{f}(x), x\right) \leq D_{f}(p, x) .
$$

In addition, by Reich and Sabach [24], if $f$ is uniformly Fréchet differentiable and bounded on bounded subsets of $X$, then we see that $F\left(\operatorname{Res}_{G}^{f}\right)=\hat{F}\left(\operatorname{Res}_{G}^{f}\right)=E P(G)$ is closed and convex. Hence, by replacing $T=\operatorname{Res}_{G}^{f}$ in Theorem 3.1, we obtain the following result. 
Theorem 4.1 Let $D, X,\left\{\alpha_{n}\right\},\left\{\beta_{n}\right\}$, and $f$ be the same as in Theorem 3.1. Let $\left\{G_{i}: D \times D \rightarrow\right.$ $R\}$ be a countable families of bifunction which satisfies the conditions (A1)-(A4) such that $E P\left(G_{i}\right) \neq \emptyset$. Let $\operatorname{Res}_{G_{i}}^{f}(x): D \rightarrow 2^{D}, i=1,2, \ldots$, be the family of mappings defined by

$$
\operatorname{Res}_{G_{i}}^{f}(x)=\left\{z \in D: G_{i}(z, y)+\langle\nabla f(z)-\nabla f(x), y-z\rangle \geq 0, \forall y \in D\right\}, \quad x \in X .
$$

Let the sequence $\left\{x_{n}\right\}$ be defined by

$$
\left\{\begin{aligned}
x_{1} \in X \quad \text { is arbitrary; } \quad D_{1}=D, & \\
G_{i}\left(w_{n, i}, y\right)+\left\langle\nabla f\left(w_{n, i}\right)-\nabla f\left(x_{n}\right), y-w_{n, i}\right\rangle \geq 0, \quad \forall y \in D, i \geq 1, & y_{n, i}=\nabla f^{*}\left[\alpha_{n} \nabla f\left(x_{1}\right)+\left(1-\alpha_{n}\right)\left(\beta_{n} \nabla f\left(x_{n}\right)\right.\right. \\
& \left.\quad\left(1-\beta_{n}\right) \nabla f\left(w_{n, i}\right)\right], \quad w_{n, i} \in \operatorname{Res}_{G_{i}}^{f}\left(x_{n}\right), i \geq 1, \\
D_{n+1}= & \left\{z \in D_{n}: \sup _{i \geq 1} D_{f}\left(z, y_{n, i}\right) \leq \alpha_{n} D_{f}\left(z, x_{1}\right)+\left(1-\alpha_{n}\right) D_{f}\left(z, x_{n}\right)+\xi_{n}\right\}, \\
x_{n+1}= & P_{D_{n+1}}^{f} x_{1} \quad(n=1,2, \ldots),
\end{aligned}\right.
$$

where $\xi_{n}=\left(k_{n}-1\right) \sup _{p \in \mathcal{F}} \zeta\left(D_{f}\left(p, x_{n}\right)\right), \mathcal{F}=\bigcap_{i=1}^{\infty} F\left(\operatorname{Res}_{G_{i}}^{f}\right), P_{D_{n+1}}^{f}$ is the Bregman projection of $X$ onto $D_{n+1}$. If $\mathcal{F}$ is nonempty, then $\left\{x_{n}\right\}$ converges strongly to $P_{\mathcal{F}}^{f} x_{1}$, which is a common solution of the system of equilibrium problems for $G_{m}, m=1,2, \ldots$.

\section{Competing interests}

The authors declare that they have no competing interests.

\section{Authors' contributions}

All authors conceived of the study, participated in its design and coordination, drafted the manuscript, participated in the sequence alignment, and read and approved the final manuscript.

\section{Acknowledgements}

The authors are very grateful to both reviewers for careful reading of this paper and for their comments.

Received: 8 May 2014 Accepted: 14 August 2014 Published: 02 Sep 2014

\section{References}

1. Chang, SS, Wang, L, Wang, XR, Chan, CK: Strong convergence theorems for Bregman totally quasi-asymptotically nonexpansive mappings in reflexive Banach spaces. Appl. Math. Comput. (2013). doi:10.1016/j.amc.2013.11.074

2. Chang, SS, Wang, L: Strong convergence theorems for Bregman totally quasi-asymptotically nonexpansive mappings in reflexive Banach spaces. Appl. Math. Comput. 228, 38-48 (2014)

3. Suthep, S, Yeol, JC, Prasit, C: Halpern's iteration for Bregman strongly nonexpansive mappings in reflexive Banach spaces. Comput. Math. Appl. 64, 489-499 (2012)

4. Li, Y, Liu, HB, Zheng, K: Halpern's iteration for Bregman strongly nonexpansive multi-valued mappings in reflexive Banach spaces with applications. Fixed Point Theory Appl. 2013, 197 (2013)

5. Reich, S, Sabach, S: Two strong convergence theorems for Bregman strongly nonexpansive operators in reflexive Banach spaces. Nonlinear Anal. 73, 122-135 (2010)

6. Nilsrakoo, W, Saejung, S: Strong convergence theorems by Halpern-Mann iterations for relatively nonexpansive mappings in Banach spaces. Appl. Math. Comput. 217(14), 6577-6586 (2011)

7. Qin, XL, Cho, YJ, Kang, SM, Zhou, HY: Convergence of a modified Halpern-type iterative algorithm for quasi- $\boldsymbol{\phi}$-nonexpansive mappings. Appl. Math. Lett. 22, 1051-1055 (2009)

8. Wang, ZM, Su, YF, Wang, DX, Dong, YC: A modified Halpern-type iteration algorithm for a family of hemi-relative nonexpansive mappings and systems of equilibrium problems in Banach spaces. J. Comput. Appl. Math. 235, 2364-2371 (2011)

9. Su, YF, Xu, HK, Zhang, X: Strong convergence theorems for two countable families of weak relatively nonexpansive mappings and applications. Nonlinear Anal. 73, 3890-3906 (2010)

10. Halpern, B: Fixed points of nonexpanding maps. Bull. Am. Math. Soc. 73, 957-961 (1967)

11. Reich, S: Strong convergence theorems for resolvents of accretive operators in Banach spaces. J. Math. Anal. Appl. 75 287-292 (1980)

12. Reich, S: Approximating fixed points of nonexpansive mappings. Panam. Math. J. 4, 23-28 (1994)

13. Rockafellar, RT: Level sets and continuity of conjugate convex functions. Trans. Am. Math. Soc. 123, 46-63 (1966)

14. Hiriart-Urruty, JB, Lemaréchal, C: Convex Analysis and Minimization Algorithms II: Advanced Theory and Bundle Methods. Grundlehren der mathematischen Wissenschaften, vol. 306. Springer, Berlin (1993) 
15. Zǎlinescu, C: Convex Analysis in General Vector Spaces. World Scientific, River Edge (2002)

16. Asplund, E, Rockafellar, RT: Gradients of convex functions. Trans. Am. Math. Soc. 139, 443-467 (1969)

17. Bonnans, JF, Shapiro, A: Perturbation Analysis of Optimization Problems. Springer, New York (2000)

18. Bauschke, HH, Borwein, JM, Combettes, PL: Essential smoothness, essential strict convexity and Legendre functions in Banach spaces. Commun. Contemp. Math. 3, 615-647 (2001)

19. Bauschke, HH, Borwein, JM, Combettes, PL: Bregman monotone optimization algorithms. SIAM J. Control Optim. 42 596-636 (2003)

20. Reich, S, Sabach, S: A strong convergence theorem for a proximal-type algorithm in reflexive Banach spaces. J. Nonlinear Convex Anal. 10, 471-485 (2009)

21. Bregman, LM: The relaxation method for finding the common point of convex sets and its application to the solution of problems in convex programming. USSR Comput. Math. Math. Phys. 7, 200-217 (1967)

22. Butnariu, D, Resmerita, E: Bregman distances, totally convex functions and a method for solving operator equations in Banach spaces. Abstr. Appl. Anal. 2006, Article ID 84919 (2006)

23. Butnariu, D, lusem, AN: Totally Convex Functions for Fixed Points Computation and Infinite Dimensional Optimization. Kluwer Academic, Dordrecht (2000)

24. Reich, S, Sabach, S: Existence and approximation of fixed points of Bregman firmly nonexpansive mappings in reflexive Banach spaces. In: Fixed-Point Algorithms for Inverse Problems in Science and Engineering. Springer, New York (2011)

25. Goebel, K, Kirk, WA: A fixed point theorem for asymptotically nonexpansive mappings. Proc. Am. Math. Soc. 35, 171-174 (1972)

26. Martín-Márquez, V, Reich, S, Sabach, S: Iterative methods for approximating fixed points of Bregman nonexpansive operators. Discrete Contin. Dyn. Syst. 6, 1043-1063 (2013)

27. Kohsaka, F, Takahashi, W: Proximal point algorithms with Bregman functions in Banach spaces. J. Nonlinear Convex Anal. 6, 505-523 (2005)

28. Zhu, JH, Chang, SS: Halpern-Mann's iterations for Bregman strongly nonexpansive mappings in reflexive Banach spaces. J. Inequal. Appl. 2013, 146 (2013)

29. Rockafellar, RT: Convex Analysis. Princeton University Press, Princeton (1970)

30. Bauschke, HH, Borwein, JM: Legendre functions and the method of random Bregman projections. J. Convex Anal. 4, 27-67 (1997)

31. Alber, YI: Metric and generalized projection operators in Banach spaces: properties and applications. In: Kartsatos, AG (ed.) Theory and Applications of Nonlinear Operator of Accretive and Monotone Type. Dekker, New York (1996)

32. Censor, Y, Lent, A: An iterative row-action method for interval convex programming. J. Optim. Theory Appl. 34, 321-353 (1981)

33. Phelps, RP: Convex Functions, Monotone Operators, and Differentiability, 2nd edn. Lecture Notes in Mathematics. Springer, Berlin (1993)

34. Li, C, Song, W, Yao, JC: The Bregman distance approximate compactness and convexity of Chebychev sets in Banach spaces. J. Approx. Theory 162, 1128-1149 (2010)

35. Maingé, PE: The viscosity approximation process for quasi-nonexpansive mappings in Hilbert spaces. Comput. Math. Appl. 59, 74-79 (2010)

36. Xu, HK: An iterative approach to quadratic optimization. J. Optim. Theory Appl. 116, 659-678 (2003)

37. Ambrosetti, A, Prodi, G: A Primer of Nonlinear Analysis. Cambridge University Press, Cambridge (1993)

38. Combettes, PL, Hirstoaga, SA: Equilibrium programming in Hilbert spaces. J. Nonlinear Convex Anal. 6, 117-136 (2005)

39. Reich, S: A Weak Convergence Theorem for the Alternating Method with Bregman Distances. Theory and Applications of Nonlinear Operators of Accretive and Monotone Type. Dekker, New York (1996)

40. Borwein, JM, Reich, S, Sabach, S: A characterization of Bregman firmly nonexpansive operators using a new monotonicity concept. J. Nonlinear Convex Anal. 12, 161-184 (2011)

41. Martín-Márquez, V, Reich, S, Sabach, S: Right Bregman nonexpansive operators in Banach spaces. Nonlinear Anal. 75, 5448-5465 (2012)

42. Martn-Mrquez, V, Reich, S, Sabach, S: Bregman strongly nonexpansive operators in reflexive Banach spaces. J. Math Anal. Appl. 400, 597-614 (2013)

10.1186/1687-1812-2014-186

Cite this article as: Li and Liu: Strong convergence of hybrid Halpern iteration for Bregman totally quasi-asymptotically nonexpansive multi-valued mappings in reflexive Banach spaces with application. Fixed Point Theory and Applications 2014, 2014:186 